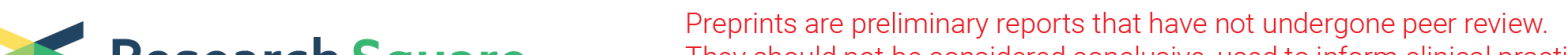 Research Square They should not be considered conclusive, used to inform clinical practice, or referenced by the media as validated information.
}

\section{Effects of exosomes from human umbilical cord mesenchymal stem cell on cardiac function in rats with dilated cardiomyopathy induced by Adriamycin}

shuangshuang liu

Qingdao Women and Children's Hospital https://orcid.org/0000-0001-9022-3774

Shun Yi

Qingdao Municipal Hospital Group

Benzhen Wang

Qingdao Women and Childrens Hospital

Chenggang Mao

the Affiliared Hospital of Qingdao University

Jingwei Chi

the Affiliated hospital of qingdao university

Kui Che

the Affiliated hospital of qingdao university

Jinmei Shen

Qingdao Women and Childrens Hospital

Xiaoqiong Lu

Qingdao Women and Childrens Hospital

Zipu Li ( $\sim$ lizipuu@163.com )

Qingdao Women and Childrens Hospital https://orcid.org/0000-0002-5244-272X

\section{Research}

Keywords: human umbilical cord mesenchymal stem cell-derived exosomes (hucMSC-Ex), dilated cardiomyopathy (DCM), tail vein injection, Cardiac function,fibrosis

Posted Date: June 5th, 2020

DOl: https://doi.org/10.21203/rs.3.rs-33077/v1

License: (c) (1) This work is licensed under a Creative Commons Attribution 4.0 International License.

Read Full License 


\section{Abstract \\ Background}

Dilated cardiomyopathy (DCM) is one of the most common cardiomyopathies in children, but we have no effective method to treat it. In recent years,the application of human umbilical cord mesenchymal stem cell (hucMSC) therapy in DCM has made a great progress, whileits mechanism remains unknown. The paracrine of hucMSCs has attracted attention as the main mechanism. In this study we explore the effect of the human umbilical cord mesenchymal stem cell-derived exosomes (hucMSC-Ex) on the cardiac function in rat models with DCM that is induced by Adriamycin.

\section{Methods}

Sprague-Dawley rats were intraperitoneally injected with Adriamycin to establish aDCM model. HucMSCEx were injected through the tail vein in the DCM group and phosphate-buffered saline (PBS) was injected in theDCMcontrolgroup.The rats were monitored for 2 weeks and thecardiac function was evaluated using thecardiac ultrasound. The pathological changes of the myocardium were observed usingimmunohistochemistry. Western blot and real-time fluorescent quantitative PCR were used to detect the expression of Smad3, alpha smooth muscle actin (a-SMA) and I type collagen (COLI)in the myocardial tissue amongthe groups of rats.Finally, the ultrastructure of the myocardium was observed using electron microscopy. High throughput sequencing was performed on the ventricular muscles in each group.

\section{Result}

High-throughput sequencing results show that after the treatment with hucMSC-Ex, the level of myocardial Scn5a in the myocardium significantly increased $(P<0.05)$. RT-PCR shows that the level of Lmod2 also significantly increased. The expression of Smad3,a-SMA and COL I was reduced $(\mathrm{P}<$ 0.05).The inflammation in the myocardial tissue decreased and the mitochondrial swelling was reduced.The left ventricular ejection function (LVEF) and left ventricular fractional shortening (LVFS)in the rats significantly increased $(P<0.05)$ and the cardiac function was significantly improved $(P<0.05)$.

\section{Conclusion}

The treatment with hucMSC-Ex through the tail vein can significantly improve thecardiac function and reduce the degree of myocardial fibrosis induced by Adriamycin.

\section{Background}


Dilated cardiomyopathy (DCM), characterized by impaired left ventricular or bilateral ventricular dilation and systolic function, is a common myocardial disease that result in heart failure [1].The incidence of cardiomyopathy in children is 1 to 2 per 100,000 people, and DCM accounts for more than half of them. Unfortunately, the transplantation rate or mortality rate of DCM in 1 or 5 years is $31 \%$ and $46 \%$,respectively,mainly due to the lack of good treatment methods [2].

Heart transplants are still the ultimate treatment method for DCM, butit is limited due to donor deficiency and immune rejection. In recent years, cell therapy has attracted more and more attention. Srauer BE et al. performed coronary reinfusion of human autologous bone marrow mononuclear cells, and the results were superior to that of the traditional standard therapies in the treatment of myocardial infarction [3]. This is the first study of stem cells in the disease treatment. However, with the development of stem cell research, the low transplanting rate, tumorigenicity and the ethics of stem cell transplantation have been widely discussed.Our previous studies found that intramuscular injection of hucMSCs improved the cardiac function in Adriamycin-induced DCM rats, while the transplanted hucMSCs were mainly retained in the muscles,meanwhile the levels of HGF,IGF-1,LIF,GM-CSF and VEGF increased in the myocardium and circulation. The results suggested that paracrine mechanisms may contribute to the therapeutic effect of hucMSCs on DCM[4].Fang et al. found that hucMSC-Ex can reduce the scar formation in wound healing by inhibiting the TGF-b1/SAMD2 pathway [5].Other studies have demonstrated that both MSC and MSCEx can reduce the SCl-induced A1 astrocytes by inhibiting the nuclear transport of NF Bp65, thus playing an anti-inflammatory and neuroprotective role [6], but the specific mechanism remains unknown.

In this study, we found that the levels of Lmod2, Scn5a and $\mathrm{Na}_{\mathrm{v}} 1.5$ has significantly increased in the myocardium of Adriamycin-induced DCM rats through the tail injection of hucMSC-Ex, and the cardiac function of DCM rats was significantly improved.In addition,we also found that hucMSC-Ex injection reduced the damage of muscle membrane and mitochondria caused by DCM and reducedthe fibrosis levels in the DCMrats' myocardium including the a-SMA, Smad3 and COLI.This study further confirmed the paracrine effect of hucMSCs, providing theoretical support for the hucMSC-Ex treatment in DCM.

\section{Methods}

Establishment of DCM rat model Male Sprague-Dawley rats (SYXK lu 20150003) aged 6 weeks were purchased from Jinan Pengyue Experimental Animal Breeding Co. (Jinan, Shandong, China), kept in the SPF animal feeding center of the Affiliated Hospital of Qingdao University the and obtain the approval of the Affiliated Hospital of Qingdao University Medical Ethics Committee. The rats in the DCM group were given intraperitoneal injection of Adriamycin (batch no. H33021980, $9 \mathrm{~g} / \mathrm{L}$ saline diluted to $1 \mathrm{~g} / \mathrm{L}$ ) of 1 $\mathrm{mg} / \mathrm{kg}$ twice a week for 8 weeks, while the rats in the normal group were intraperitoneally injected with the same volume of saline $9 \mathrm{~g} / \mathrm{L}$ twice a week for 8 weeks. Echocardiography was used to assess the success of the model. The rats were kept in comfortable environments, including comfortable cages, with appropriate temperature and light and free access to water and food. 
During the modeling process, the rats were continuously observed.In the normal group, all the rats showed normal daily activities and normal growth without death incidents, while in the DCM group, 34 out of 40 rats (85\%) survived for 8 weeks after modeling.In addition, the surviving rats showed loss of appetite, decreased exercise, slow reaction, slow growth, severe depilation and ascites.

Preparation of hucMSCs The umbilical cord was obtained from a healthy newborn baby immediately after birth with the full informed consent of its legal guardian and the approval of the Affiliated Hospital of Qingdao University Medical Ethics Committee.Screening was done for human immunodeficiency virus $(\mathrm{HIV})$, hepatitis $\mathrm{C}$ virus $(\mathrm{HCV})$, cytomegalovirus (CMV), hepatitis $\mathrm{B}$ virus (HBV) and treponema pallidum. Huatong glue from the umbilical cord was extracted and the organization block adherent method was used to cultivate hucMSCs, and subculture, in appropriate algebra cells cryopreserved in liquid nitrogen.

Extraction of hucMSC-Ex The 3rd generation hucMSCs with good growth status were resuscitated and screened for aerobic bacteria, mycoplasma, HIV, HBV, HCV, CMV and endotoxin. The morphology and immunophenoype of the cells were examined (CD34, CD44, CD45, CD146, CD105 and HLA-DR). The supernatants were collected after $48 \mathrm{~h}$ of starvation, and the purified exosomes were obtained using ultrahigh-speed centrifugation. Finally, the exosomes were resuspended by PBS.

Identification of hucMSC-Ex The morphology of the exosomes was observed using the transmission electron microscopy. Western blot was used to detect the exosomes' specific markers CD63, CD81 and CD9 and the particle size of the exosomes was determined by NTA.

HucMSC-Ex treatment Two weeks after the establishment of the DCM rat model, the surviving DCM rats were randomly divided into two groups (17 in each group): the DCM control group that was injected with $2.0 \mathrm{~mL}$ PBS once a week, and the hucMSC-Ex group that was injected with $250 \mathrm{ug} / \mathrm{kg}$ of hucMSC-Ex through the tail vein once a week.The general condition of the rats was observed 24 hours after the injection to observe the adverse effects. This initial treatment was followed for 4 weeks.

Echocardiography Before initiating the hucMSC-Ex injection and 2 weeks after the final treatment, the heart was explored by M-mode echocardiography using the Philips iE33 xMATRIX echocardiography system (Philips Healthcare, Amsterdam, Netherlands) that is equipped with 8-12 MHz ultrasound probe. The parameters related to the cardiac function were measured and calculated,including left ventricular inner systolic diameter (LVIDs), left ventricular inner diastolic diameter (LVIDd), left ventricular ejection function (LVEF) and left ventricular fractional shortening (LVFS).

Immunohistochemistry Two weeks after the final treatment, the rats were euthanized by intraperitoneal injection of a lethal dose of chloral hydrate solution $(200 \mathrm{mg} / \mathrm{kg})$. The ventricular muscle tissues of the rats were then taken to be fixed with paraformaldehyde, embedded in paraffin, sliced, incubated with antibodies, stained and observed under an optical microscope (Olympus BX51, Tokyo, Japan).

Western blot After the rats were euthanized,we collect their ventricular muscle tissue. Western blot was used to detect their ventricular muscle tissueCOLI, Smad3 and a-SMA protein expression and calculate 
the relative expression quantity.

Real-time fluorescent quantitative PCR After collecting the ventricular muscle tissue of the euthanized rats,total RNA was extracted and then resuspended in DEPC- $\mathrm{H}_{2} \mathrm{O}$.The PrimeScript RT reagent Kit with gDNA Eraser(Takara, Japan) was used to reverse transcribe the total extracted RNA to produce cDNA. Using the TB Green ${ }^{\mathrm{TM}}$ Premix Ex Taq ${ }^{\mathrm{TM}}{ }_{\mathrm{II}}$ (TliRNaseH Plus) (Takara, Roche cobasz480) for real-time quantitative $\mathrm{CDNA}$, the quantitative process involved $95^{\circ} \mathrm{C} 30 \mathrm{~s}$ for one cycle, $95^{\circ} \mathrm{C}$ s and $30 \mathrm{~s} 60^{\circ} \mathrm{C}$ for a total of 30 cycle.The GAPDH gene was used as the normalization control. The cycle number at the fluorescence threshold (CT) was used as an indicator of the expression level of the target gene. The expression levels in the normal controls were used as the comparative reference, and relative quantification of the expression in the study groups was determined by the $2^{-\Delta \Delta C T}$ values. The primers that were used in the process were designed and synthesized by the Huada gene company.

Electron microscope Two weeks after the final treatment, the rat myocardial tissue was fixed, embedded, sectioned and stained;the ultrastructure of the myocardium was then observed by the JEOL jem-1200ex transmission electron microscopy.

Statistical analysis Statistical analyses were performed using the SPSS 19.0 software (SPSS Inc., Chicago, IL, USA). Data were expressed in the form of mean \pm SEM. We performed one-way ANOVA in conjunction with the least significant difference test to determine the statistical significance $(P<0.05)$.

\section{Results}

\section{Effect of hucMSC-Ex on the survival of DCM rats}

The survival rates of the rats in the normal group, DCM group and hucMSC-Ex group were 100\% (20/20), $58.82 \%(10 / 17)$ and $82.35 \%(14 / 17)$, respectively. The treatment with hucMSCs-Ex could improve the appetite and exercise of DCM rats, and reduce the ascites and depilation, that were persistent in DCM rats before and after the treatment.No local inflammation, ulcers or induration were detected at the injection site. The postmortem examination of the dead rats during the hucMSCs-Ex treatment showed hepatosplenomegaly, bloody ascites, and pleural effusion,these results suggest possible death due to heart failure complications.

\section{Identification of hucMSCs and hucMSC-Ex}

Under optical microscope, the hucMSCs cells were spindle-shaped or polygonal and grew up like vortex(Additional file 1: Fig. 3A).The flow cytometry results showed that the hucMSCs specific surface markers CD44 and CD105 were positive, but the hematopoietic and endothelial cell specific markers CD34, CD45 and HLA-DR were negative(Additional file 1: Fig. 3B). We extracted hucMSC-Ex from the hucMSCs and its high expression of CD81, CD9 and TSG101 proteins was identified using western blot(Fig.1a). The transmission electron microscopy (TEM) showed that the diameter was mainly around 40-100nm, and the internal vesicles contained a high-density electronic dense matter(Fig.1b).The results 
of NTA showed that the diameter of the exosomes ranged from $40 \mathrm{~nm}$ to $100 \mathrm{~nm}$, with a peak at 100nm(Fig.1c).

\section{Effects of hucMSC-Ex on the cardiac function in DCM rats}

Before the hucMSC-Ex treatment, there was no difference in the heart status among all rats in the DCM groups, with the ventricular cavity size (represented by LVIDs and LVIDd) being significantly enlarged compared with the normal group and the cardiac function (represented by LVEF and LVFS) being significantly decreased compared with the normal group(Fig.2a,detailed data were not listed).LVEF and LVFS were significantly higher after the four-weeks treatment with hucMSC-Ex compared with the DCM control group(Fig. 2b). There was no significant change in LVIDs and LVIDd compared with the DCM control group after the hucMSC-Ex treatment (detailed data were not listed).

\section{Effects of hucMSCs-Ex on the expression of cardiac cytokines in DCM rats}

Real time quantitative PCR results showed that compared with the normal control rats, the level of Lmod2 and Scn5a was reduced in all DCM rats, while the level of Lmod2 and Scn5a was significantly increased after the hucMSC-Ex treatment compared with the DCM rats (Fig.3a-b). Compared with the normal control rats, the level of a-SMA was increased while it was decreased after the hucMSC-Ex treatment(Fig.3c).Western blot test results showed that compared with the normal control rats, the expression of $\mathrm{Na}_{\mathrm{v}} 1.5$ in DCM rats was decreased, and it was increased after the hucMSC-Ex treatment(Fig.3d).Additionally, compared with normal group, the expression of COL $\otimes$, Smad3 and a-SMA was increased and it was significantly reduced after the hucMSC-Ex treatment(Fig.3e-g).

\section{Effects of hucMSCs-Ex on the myocardial tissue structure in DCM rats}

HE staining showed that in the normal control group, the myocardial fibers were orderly arranged, the nuclei were lightly stained, the cells were not degenerated or necrotic and the interstitium was not infiltrated by bleeding or inflammatory cells(Fig.4a). On the other hand, the myocardial fibers in the DCM group were arranged in disorder with rupture and wavy arrangement. There were interstitial edema, inflammatory cell infiltration and bleeding among the myocardial fibers(Fig.4b-c).After treatment with hucMSC-Ex, the myocardial cell injury was significantly reduced(Fig.4d).

The degree of myocardial fibrosis was evaluated using Masson's staining, and the volume fraction of collagen was measured (CVF = collagen area/visual field area $\times 100 \%$ ).Compared with the normal group(Fig.5a-b), Masson's staining of the DCM rats'myocardial tissue showed a large amount of bluestained collagen deposition between and around the heart muscle tissue (Fig.5c-d). Compared with the DCM rats, the deposition of fibrous tissue was significantly decreased after treatment with hucMSCsEx(Fig.5e-f), and the CVF was also significantly decreased(Additional file 1:Fig. S4 B).

The ultrastructure of the cardiomyocytes was observed using the transmission electron microscopy, and it was found that the myolemma of the cardiomyocytes in the normal control rats was intact, and the 
myocardial fibers were arranged in an orderly manner, showing clear Z-line and H-zone. The mitochondria were oval-shaped and linear with no absence, swelling,vacuoles,or dense electron deposits(Fig.6a).In the DCM rats, the myolemma of the myocardial cells was lysed, the myofibrils were atrophy, disordered and intercellular space widens, the myofilaments were broken in some areas and their structures were fuzzy, the mitochondria were swollen with vacuolar degeneration, and cristae structures were absent. There were alsoglycogen deposits between the myofibrils in some areas(Fig.6b-C).After treatment with hucMSCEx, the cardiomyocytes maintained the integrity of the myolemma to a large extent, and the myofibrils were arranged in an orderly manner with clear structure.Most of the mitochondria remained elliptic and partially linear, with no vacuolar degeneration and almost complete cristae despite mild swelling(Fig .6d).

High-throughput sequencing of myocardial tissue High-throughput shows that the DCM rats had 965 upregulated genes and 810 down-regulated genes compared with the normal control rats, while hucMSCsEx treatment led to 321 up-regulated genes and 375 down-regulated ones compared with the DCM rats(Fig.7a-b).

\section{Discussion}

In recent years, hucMSCs[7,8], which have the function of promoting tissue repair and angiogenesis, have become an effective alternative to treat DCM. However, both coronary administration and intramuscular injection have the disadvantages of trauma and the retention of hucMSCs in the muscles, which greatly limits the extensive clinical application of hucMSCs. Our previous studies have also found that hucMSCs may act through paracrine. In consistence with our findings, Huang et al. found that hucMSCs can promote the myocardial microangiogenesis, inhibit the myocardial fibrosis by secreting effective biosignaling molecules such as cytokines, improve the myocardial remodeling, prevent the myocardial cell apoptosis, reduce the oxidative stress response, and ultimately improve the cardiac function $[9,10]$.In this study, echocardiography showed that caudal administration of hucMSC-Ex increased the myocardial contractility in DCM rats and increased the expression of DCM-related genes in the myocardial tissue, including Lmod2 and Scn5a.It suggests that hucMSC-Ex may improve cardiac function by regulating the level of DCM-related genes.

Exosomes are tiny membrane vesicles with diameters of 40-100nm that are secreted by most cells in the body and contain a large amount of lipids, proteins and RNA[11], which can mediate the information transferring between cells. Our initial experiments confirmed that normal rats injected with exosomes through the tail vein did not develop abnormal cardiac function nor local inflammation or ulcers at the injection site (data not listed), this indicates that the exosomes have low immunogenicity and their injection through the tail vein is a safe treatment.

In this experiment, DCM rats developed severe ascites, delayed movement and anorexia after modeling, which were related to the decline of the cardiac function and were consistent with the clinicopathological features of DCM. We cultured hucMSCs in serum-free medium (Wuhan benover, China) to exclude the effect of the exosomes in the medium on the experimental results [12].After separation and purification, 
hucMSC-Ex with high purity were injected into DCM rats through the tail vein for 4 weeks.

Echocardiography showed that the cardiac function was significantly improved, and LVEF and LVFS were significantly increased.In addition, high-throughput sequencing of the myocardial tissue showed that the DCM rats had 965 up-regulated genes and 810 down-regulated genes compared with the normal control rats, while hucMSCs-Ex treatment led to 321 up-regulated genes and 375 down-regulated ones compared with the DCM rats. The changes of Scn5a were more significant. In addition, RT-PCR shows that the level of Lmod2 increased after the treatment with hucMSC-Ex. Lmod2 mainly plays a role in regulating the length of the muscle filaments in striated muscles, with the highest expression level in the myocardial tissue [13].Studies have shown that knocking out the Lmod2 gene in mice causes the filaments of myocardial fibers to become abnormally short, and that young mice may also die due to the cardiac systolic dysfunction and ventricular dilatation [14].In this study, it was found that the level of Lmod2 in the myocardial tissue of the DCM rats induced by Adramycin was decreased, indicating that the length of the filaments of myocardial fibers was shortened and the cardiac systolic dysfunction was observed in rats after modeling, which was consistent with the pathological mechanism of DCM. After treatment with hucMSC-Ex, the level of Lmod2 increased to the same level as that of the normal control group, and the cardiac function was significantly improved, indicating that the length of the filaments of myocardial fibers was largely recovered to the original level.Lmod2 is the first identified protein in mammals that regulates the length of striated muscle filaments, and its mutation can lead to the development of heart enlargement in children [15].SCN5a is also a pathogenic gene that is associated with familial DCM, which encodes the sodium channel protein $\left(\mathrm{Na}_{\mathrm{v}} \mathrm{1.5}\right)$ in cardiomyocytes. This channel mainly controls the inward sodium current (INa), which is the basis for the rapid rise of the cardiac action potential and plays a crucial role in regulating the electrophysiological function of the heart [16].The reduction of the ratedependent $\mathrm{Na}^{+}$current and the increase of the late sodium current (INaL) interfere with the intracellular $\mathrm{Na}^{+}$homeostasis causing DCM, such as A1180V [17].In our experiment, we found that the expression of Scn5a in the Adriamycin-induced DCM rats was decreased and the expression of $\mathrm{Na}_{\mathrm{v}} 1.5$ protein was decreased, indicating that the $\mathrm{Na}^{+}$homeostasis of the DCM rats'cardiomyocytes was unbalanced, the depolarization rate was slowed and the electrophysiology of the cardiomyocytes was disturbed. After treatment with hucMSC-Ex, the above-mentioned expression increased to the same level as that of normal control rats, which almost reversed the imbalance of $\mathrm{Na}^{+}$homeostasis in cardiomyocytes, the depolarization and repolarization of the cardiomyocytes returned to the normal level and the cardiac function was improved.

According to the experimental results, it is speculated that Lmod2 and Scn5a in hucMSC-Ex have entered into the DCM cardiomyocytes and expressed their proteins to maintain the length of the muscle filament or stabilize the internal environment of the cells by expressing the Lmod2 and $\mathrm{Na}_{\mathrm{v}} 1.5$ proteins, thus improving the cardiac function. In consistence with our hypothesis, HadiValadi et al. found that the RNA from mast cells exosomes can be transferred to other mouse and human mast cells, and new mouse proteins were found in the recipient cells which indicated that the transferred exosome mRNA can enter into another cell and can be translated [18].Ratajczak $\mathrm{J}$ et al. identified a mechanism that allows the 
exosomes derived from embryonic stem cells to accumulate large amounts of mRNA before shedding from parental stem cells [19].

In addition, our experiment also found that the DCMrats' myocardial tissue fibrosis indexes including aSMA [20], Smad3 and COL $\otimes[21]$ proteins have a significant rise. Masson staining in the DCM rats' myocardial tissue collagen content indicates that serious myocardial fibrosis has happened. After treatment with hucMSC-Ex, the expression of a-SMA, Smad3 and COL $\otimes$ proteins has been significantly reduced, and Masson staining in the myocardial tissue collagen content indicates that the myocardial fibrosis has been reduced. Therefore, hucMSC-Ex can also improve the cardiac function in DCM by regulating the fibrosis level.

In the dose range of this study, treatment with hucMSC-Ex did not result in significant changes in the size of the left ventricle. Nevertheless, our observations of the ultrastructure of the myocardial cells suggest that hucMSC-Ex injection can reduce the cellular damage caused by DCM, such as the repair of the myolemma loss, cardiomyocyte disruption and mitochondrial degeneration,thus providing a theoretical basis for hucMSC-Ex treatment of DCM at the cellular level. Higher doses and longer injections may be needed to cause the left ventricle to change in size.

There are great developmental prospects for the use of hucMSCs in improving the damaged heart muscle. Since hucMSC-Ex carry all of parental cells [18] or more of the genetic material to a large extent, it is possible that hucMSC-Ex is a kind of new feasible treatment method that doesn't need to transplant the whole cell in the injured heart tissue.In the future, our research team will conduct further experiments and analysis on hucMSC-Ex considering the improvement of cardiac function from multiple levels and perspectives, so as to provide scientific basis for the possible use of hucMSC-Ex in the clinical treatment of DCM.

\section{Conclusion}

We make DCM rats models successfully,because the ventricular cavity size (represented by LVIDs and LVIDd) being significantly enlarged compared with the normal group and the cardiac function (represented by LVEF and LVFS) being significantly decreased compared with the normal group in DCM rats.And then LVEF and LVFS were significantly higher after the four-weeks treatment with hucMSC-Ex compared with the DCM control group.These results mean that hucMSC-Ex can improve DCM rats' cardiac function.

RT-PCR results showed that compared with the normal control rats, the level of Lmod2 and Scn5a was reduced in all DCM rats, while the level of Lmod2 and Scn5a was significantly increased after the

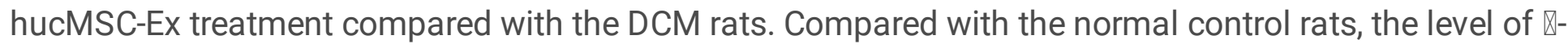
SMA was increased while it was decreased after the hucMSC-Ex treatment. These results means that hucMSC-Ex can increase DCM-related genes'level to improve DCM rats'cardiac function. 
Western blot test results showed that compared with the normal control rats, the expression of $\mathrm{Na}_{\mathrm{v}} 1.5$ in DCM rats was decreased, and it was increased after the hucMSC-Ex treatment.Additionally, compared

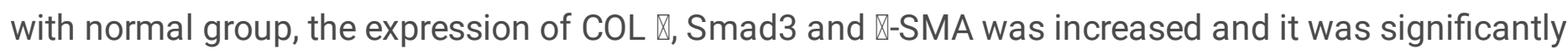
reduced after the hucMSC-Ex treatment.These results means that hucMSC-Ex can improve DCM rats'myocardial fibrosis to improve DCM rats'cardiac function.

HE staining,Masson staining and Transmission electron microscopy images of heart tissues all showed that DCM rats'myocardial inflammation,fibrosis and swelling of mitochondria decreased,which means that hucMSC-Ex can improve DCM rats'cardiac function through cellular level.

\section{Abbreviations}

DCM:dilated cardiomyopathy ; hucMSC :human umbilical cord mesenchymal stem cell human ;hucMSC-

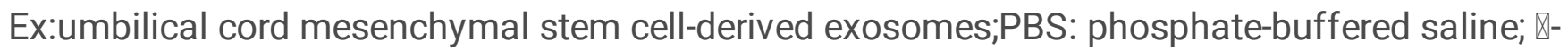
SMA:alpha smooth muscle actin; COLI :I type collagen; LVEF:left ventricular ejection function;LVFS: left ventricular fractional shortening; HIV: Human immunodeficiency virus;CMV: Cytomegalovirus; HBV: Hepatitis B virus; HCV: Hepatitis C virus; LVEF: Left ventricular ejection fraction; LVFS: Left ventricular fraction shortening;LVIDd: Left ventricular inner end-diastolic diameter; LVIDs: Left ventricular inner endsystolic diameter.

\section{Declarations}

\section{Availability of data and materials:}

All data generated or analysed during this study are included in this published article [and its additional information files]

\section{Acknowledgements:}

We thank the Department of ultrasound of the Affiliated Hospital of Qingdao University, China, for their assistance in echocardiography of rats.

\section{Funding:}

This study is funded by the Provincial Natural Science Foundation of

Shandong (\#ZR2017PH040) and the Science and Technology Support Program of Qingdao(\#14-2-3-16nsh).

\section{Authors' contributions:}

SSL and SY contributed to the study design, study performance and preparation of the manuscript. ZPL contributed to the study design, data analysis and interpretation, and preparation and revision of the 
manuscript.BZW and CGM contributed to the study design, data analysis, and preparation of the manuscript.JWC,KC and JMS contributed to the study performance.Xiaoqiong Lu contributed to the study data analysis.SSL and SY contributed equally to this work and should be considered coauthors. All authors read and approved the final manuscript.

\section{Competing interests:}

The authors declare that they have no competing interests.

\section{Consent for publication:}

Not applicable.

\section{Ethics approval and consent to participate:}

Umbilical cords were collected from healthy newborn children with the fully informed consent from their legal guardians and approval from the Medical Ethics Council of the Affiliated Hospital of Qingdao University ,and all the procedures involving the rats were approved by the Ethics Committee in Animal Research of Qingdao University according to the university regulations for animal research (QYFYWZLL25674).

\section{Authors information:}

SSL:Pediatrics, the Qingdao University Affiliated Qingdao Women and Children's Hospital, 6 Tongfu Rd, Qingdao 266011, China. Pediatrics邓the Affiliated Hospital of Qingdao University, 16 Jiangsu Rd, Qingdao 266003, China.

SY:Department of Thoracic Surgery, Qingdao Municipal Hospital(Group),1Jiaozhou Rd, Qingdao 2266011,China.

BZW:Pediatrics, the Qingdao University Affiliated Qingdao Women and Children's Hospital, 6 Tongfu Rd, Qingdao 266011, China.

CGM:Department of Pediatric Cardiology, Nephrology and Rheumatology,the Affiliated Hospital of Qingdao University, 16 Jiangsu Rd, Qingdao 266003, China.

JWC:Qingdao Key Laboratory of Thyroid Diseases, the Affiliated Hospital of Qingdao University , 16 Jiangsu Rd, Qingdao 266003, China.

KC: Qingdao Key Laboratory of Thyroid Diseases, the Affiliated Hospital of Qingdao University, 16 Jiangsu Rd, Qingdao 266003, China.

JMS: Pediatrics, the Qingdao University Affiliated Qingdao Women and Children's Hospital, 6 Tongfu Rd, Qingdao 266011, China. 
Xiaoqiong Lu, the Qingdao University Affiliated Qingdao Women and Children's Hospital, 16 Jiangsu Rd, Qingdao 266003, China.

ZPL:Pediatrics, the Qingdao University Affiliated Qingdao Women and Children's Hospital, 6 Tongfu Rd, Qingdao 266011, China.

\section{References}

1. Felix SB, Beug D, Dörr M. Immunoadsorption therapy in dilated cardiomyopathy. Expert Rev Cardiovasc Ther. 2015;13:145-152. doi: 10.1586/14779072.2015.990385.

2. Rupp S,Jux C. Advances in heart failure therapy in pediatric patients with dilated cardiomyopathy. Heart Fail Rev.2018;23:555-562.doi: 10.1007/s10741-018-9692-1.

3. Strauer BE, Brehm M, Zeus T, et al. Repair of infarcted myocardium by autologous intracoronary mononuclear bone marrow cell transplantation in humans. Circulation. 2002;106:1913-1918. doi: 10.1161/01.cir.0000034046.87607.1c.

4. Mao C, Hou X, Wang B, et al. Intramuscular injection of human umbilical cord-derived mesenchymal stem cells improves cardiac function in dilated cardiomyopathy rats. Stem Cell Res Ther. 2017;8:18. doi:10.1186/s13287-017-0472-y.

5. Fang S, Xu C, Zhang Y, et al. Umbilical Cord-Derived Mesenchymal Stem Cell-Derived Exosomal MicroRNAs Suppress Myofibroblast Differentiation by Inhibiting the Transforming Growth Factorbeta/SMAD2 Pathway During Wound Healing. Stem Cells Transl Med. 2016;5: 1425-1439. doi: 10.5966/sctm.2015-0367.

6. Wang L, Pei S, Han L, et al. Mesenchymal Stem Cell-Derived Exosomes Reduce A1 Astrocytes via Downregulation of Phosphorylated NFKB P65 Subunit in Spinal Cord Injury. Cell Physiol Biochem. 2018;50:1535-1559.doi: 10.1159/000494652.

7. Zhu J, Liu Q, Jiang Y, Wu L, Xu G, Liu X. Enhanced angiogenesis promoted by human umbilical mesenchymal stem cell transplantation in stroked mouse is Notch1 signaling associated. Neuroscience. 2015;2:290:288-99.doi: 10.1016/j.neuroscience.2015.01.038.

8. Chen Y, Yu Y, Chen L, et al. Human Umbilical Cord Mesenchymal Stem Cells: A New Therapeutic Option for Tooth Regeneration. Stem Cells Int. 2015;2015:549432.doi: 10.1155/2015/549432.

9. Huang J, Zhang Z, Guo J, et al. Genetic modification of mesenchymal stem cells overexpressing CCR1 increases cell viability, migration, engraftment, and capillary density in the injured myocardium. Circ Res.2010;106: 1753-1762.doi: 10.1161/CIRCRESAHA.

10. Lopes JP, Fiarresga A, Silva Cunha P, et al. [Mesenchymal stem cell therapy in heart disease]. Rev Port Cardiol.2013;32: 43-47. doi: 10.1016/j.vph.2012.04.002.

11. Abbaszadeh H, Ghorbani F, Derakhshani M, et al. Human umbilical cord mesenchymal stem cellderived extracellular vesicles: A novel therapeutic paradigm. J Cell Physiol, 2020 ;235:706-717.doi: 10.1002/jcp.29004. 
12. Witwer KW, Buzás El, Bemis LT, et al. Standardization of sample collection, isolation and analysis methods in extracellular vesicle research. J Extracell Vesicles.2013;27:2. doi:10.3402/jev.v2i0.20360.

13. Li S, Mo K, Tian H, et al. Lmod2 piggyBac mutant mice exhibit dilated cardiomyopathy. Cell Biosci. 2016;6:38. doi:10.1186/s13578-016-0101-y.

14. Pappas CT, Mayfield RM, Henderson C, et al. Knockout of Lmod2 results in shorter thin filaments followed by dilated cardiomyopathy and juvenile lethality. Proc Natl Acad Sci U S A. 2015;112:13573-13578.doi:10.1073/pnas.1508273112.

15. Ahrens-Nicklas RC, Pappas CT, Farman GP, et al. Disruption of cardiac thin filament assembly arising from a mutation in $<i>L M O D 2</ i>$ : A novel mechanism of neonatal dilated cardiomyopathy. Sci Adv. 2019;5:eaax2066.doi: 10.1126/sciadv.aax2066.

16. Veerman CC, Wilde AA, Lodder EM. The cardiac sodium channel gene SCN5A and its gene product NaV1.5: Role in physiology and pathophysiology. Gene.2015;573:177-

187.doi:10.1016/j.gene.2015.08.062.

17. Li W, Yin L, Shen C, Hu K, Ge J, Sun A. SCN5A Variants: Association With Cardiac Disorders. Front Physiol. 2018;9:1372. doi:10.3389/fphys.2018.01372.

18. Valadi H,Ekström K, Bossios A,Sjöstrand M,Lee JJ ,Lötvall JO.Exosome-mediated transfer of mRNAs and microRNAs is a novel mechanism of genetic exchange between cells. Nat cell Biol.2007;9:645659. doi: $10.1038 / \mathrm{ncb} 1596$.

19. Ratajczak J, Miekus K, Kucia, M. et al. Embryonic stem cell-derived microvesicles reprogram hematopoietic progenitors: evidence for horizontal transfer of mRNA and protein delivery. Leukemia.2006;20:847-856.doi:10.1038/sj.leu.2404132.

20. Meng XM, Nikolic-Paterson DJ, Lan HY. TGF-beta: the master regulator of fibrosis. Nat Rev Nephrol. 2016;12: 325-338. doi: 10.1038/nrneph.2016.48.

21. Tao G, Levay AK, Peacock JD, et al. Collagen XIV is important for growth and structural integrity of the myocardium. J Mol Cell Cardiol. 2012;53:626-38.doi: 10.1016/j.yjmcc.

\section{Figures}


a

CD9

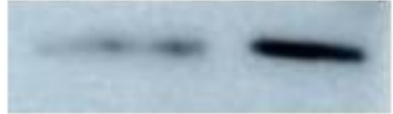

hucMSCs exosome
CD81

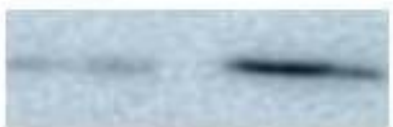

hucMSCs exosome
TSG101

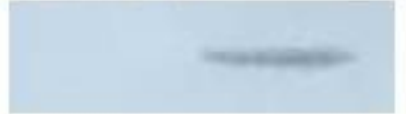

hucMSCs exosome b

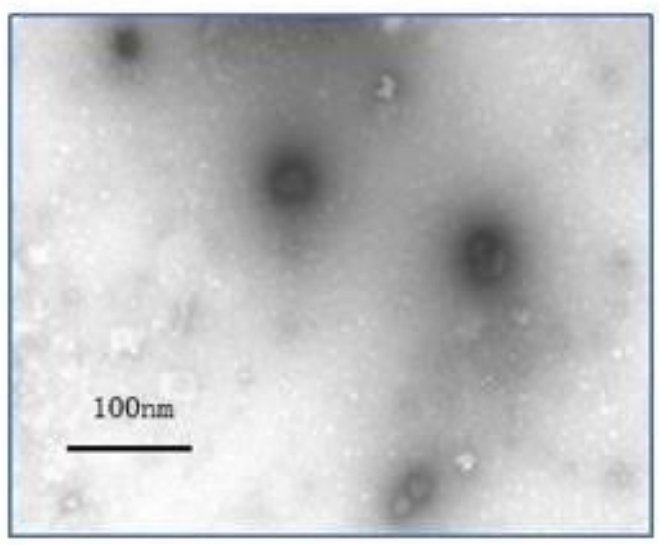

c

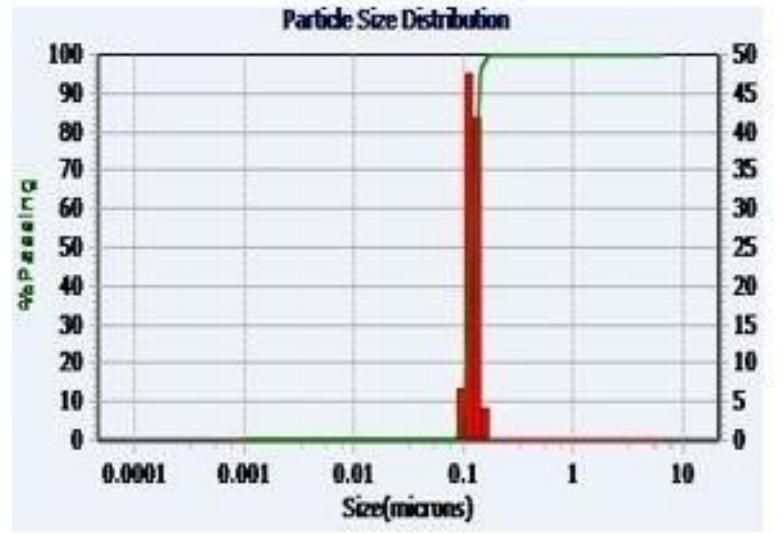

\section{Figure 1}

(a)Western blot of hucMSCs and exosome including CD9,CD81and TSG101. (b)Transmission electron microscopy of exosomes.Black bars=100nm. (c)NTA of exosomes. 
a
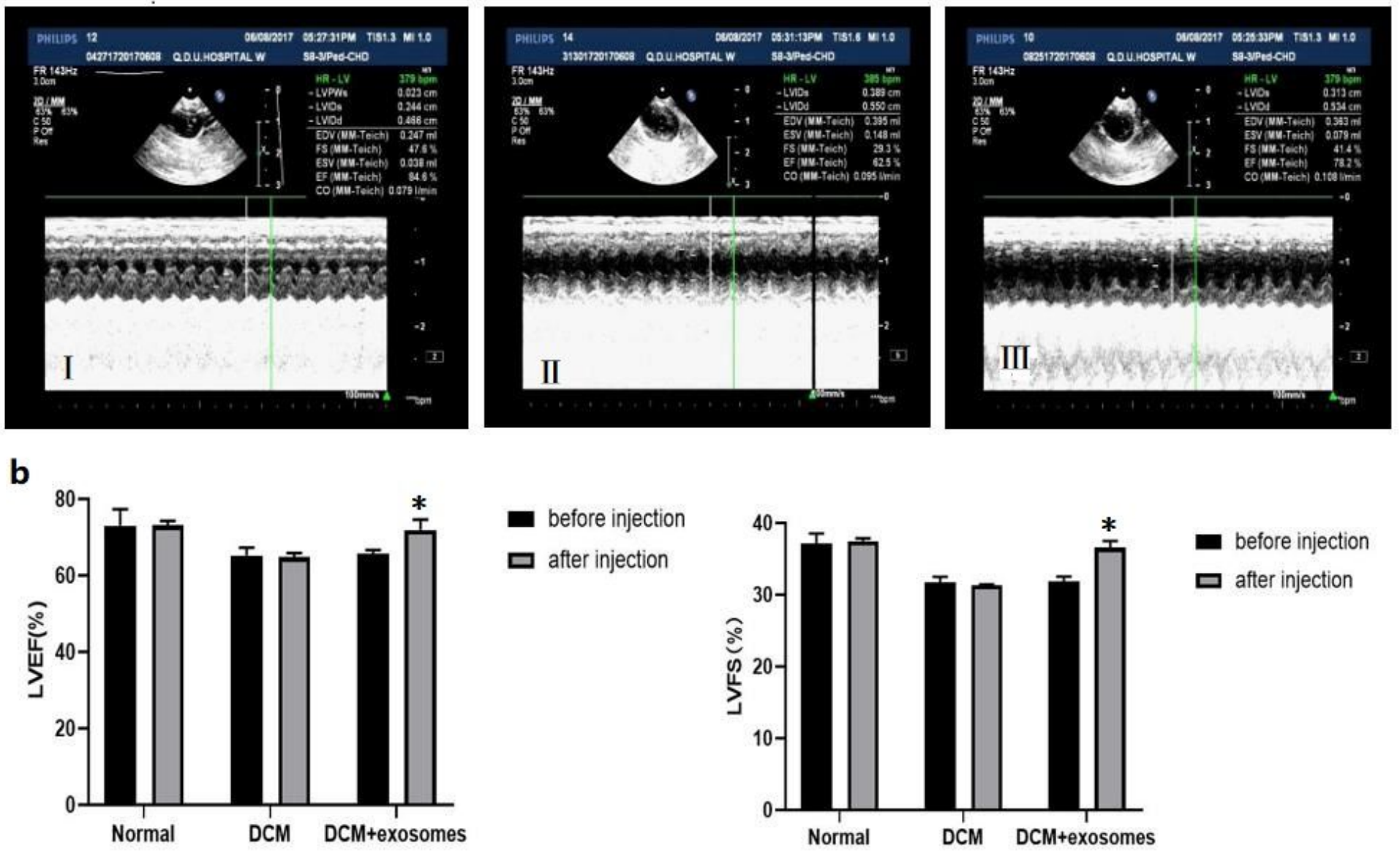

Figure 2

(a)Echocardiography of the normal control (I), DCM control (II), exosomes group (III) rats. (b) LVEF and LVFS of the normal control(Normal), DCM control (DCM), exosomes-treated (DCM+exosomes) rats.Blacksolid bars represent LVEF or LVFS before the exosomes treatment, and grey solid bars represent LVEFor LVFS after the exosomes treatment. Asterisks represent the statistical difference $(P<0.05$, ANOVA). 

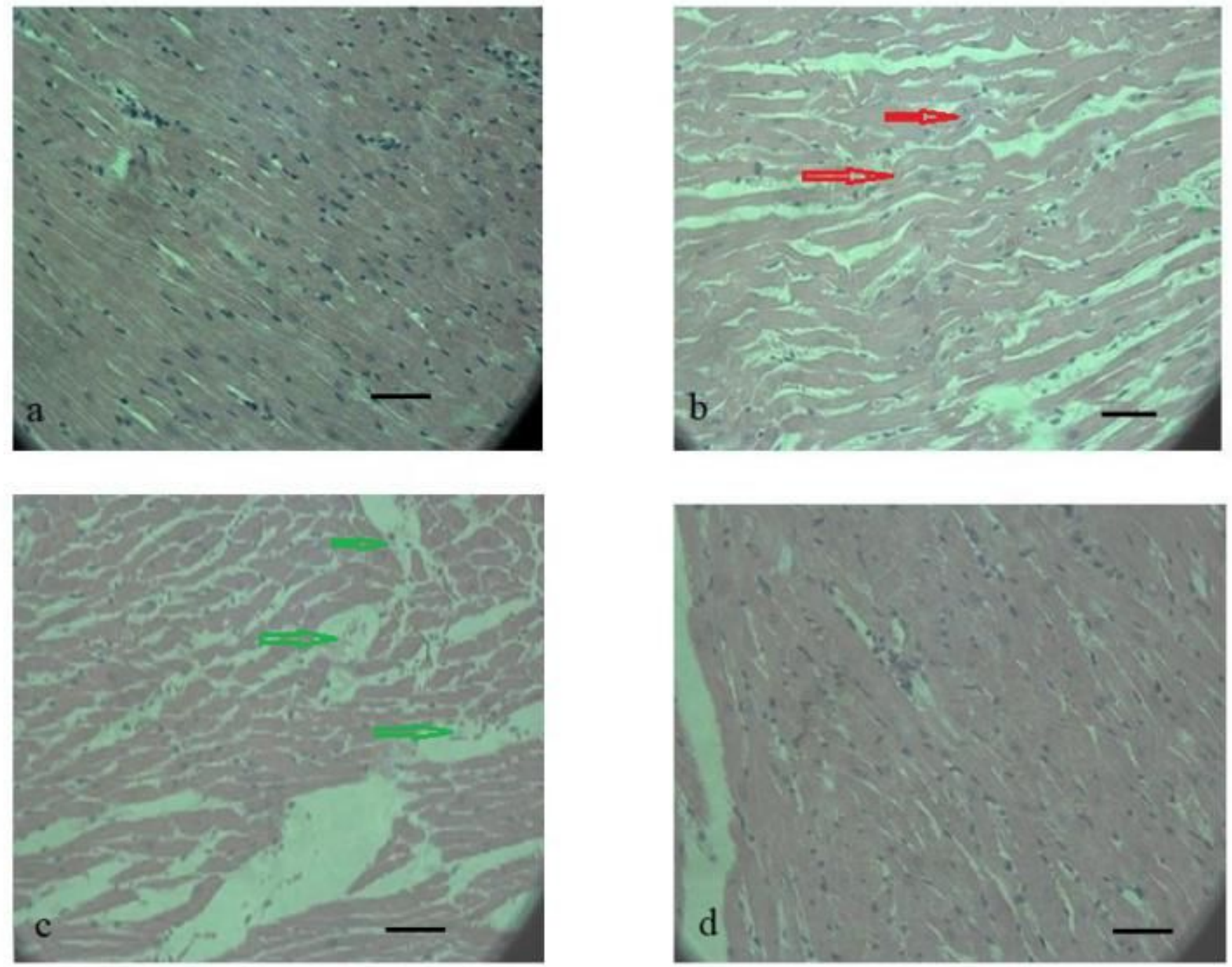

\section{Figure 3}

Hemoxylin-eosin stain of heart tissues derived from the normal control (a), the DCM control (b-c) and the exosomes group (d). The red arrows show wavy arrangement of sparse myofibrils; the green arrows show bleeding. Black bars $=50 \mu \mathrm{m}$ 

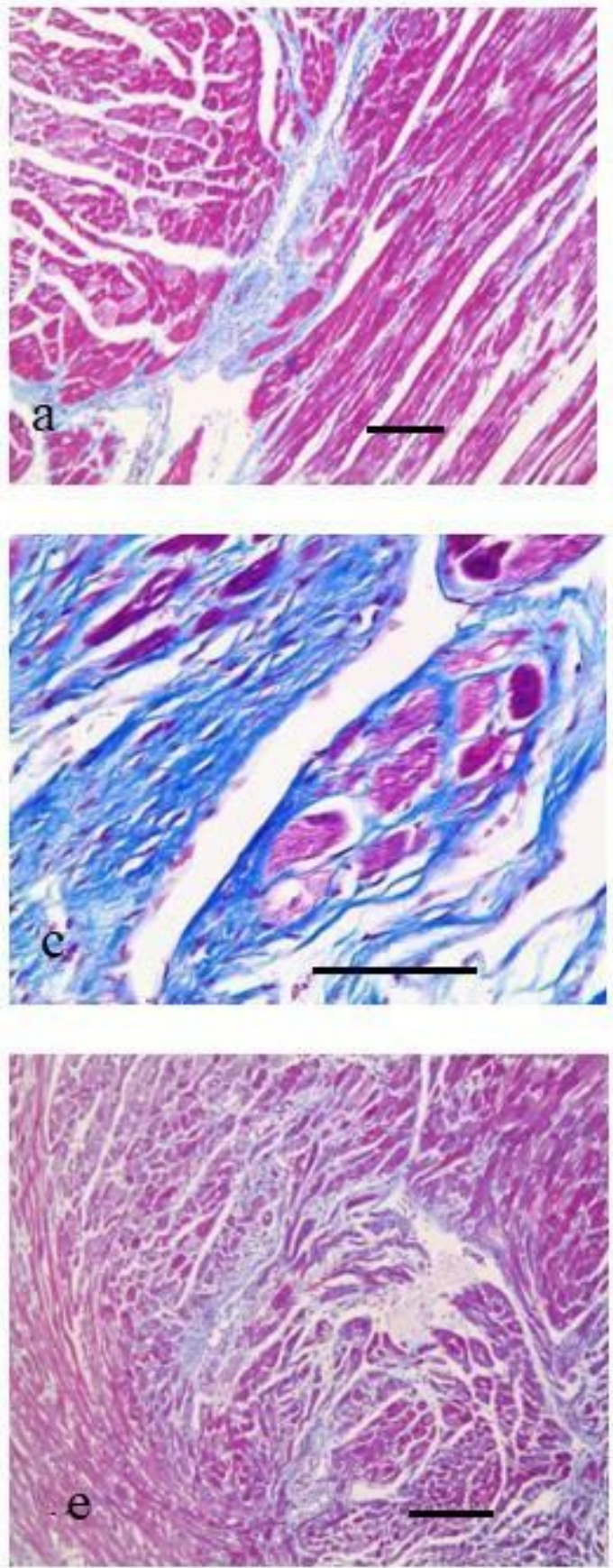
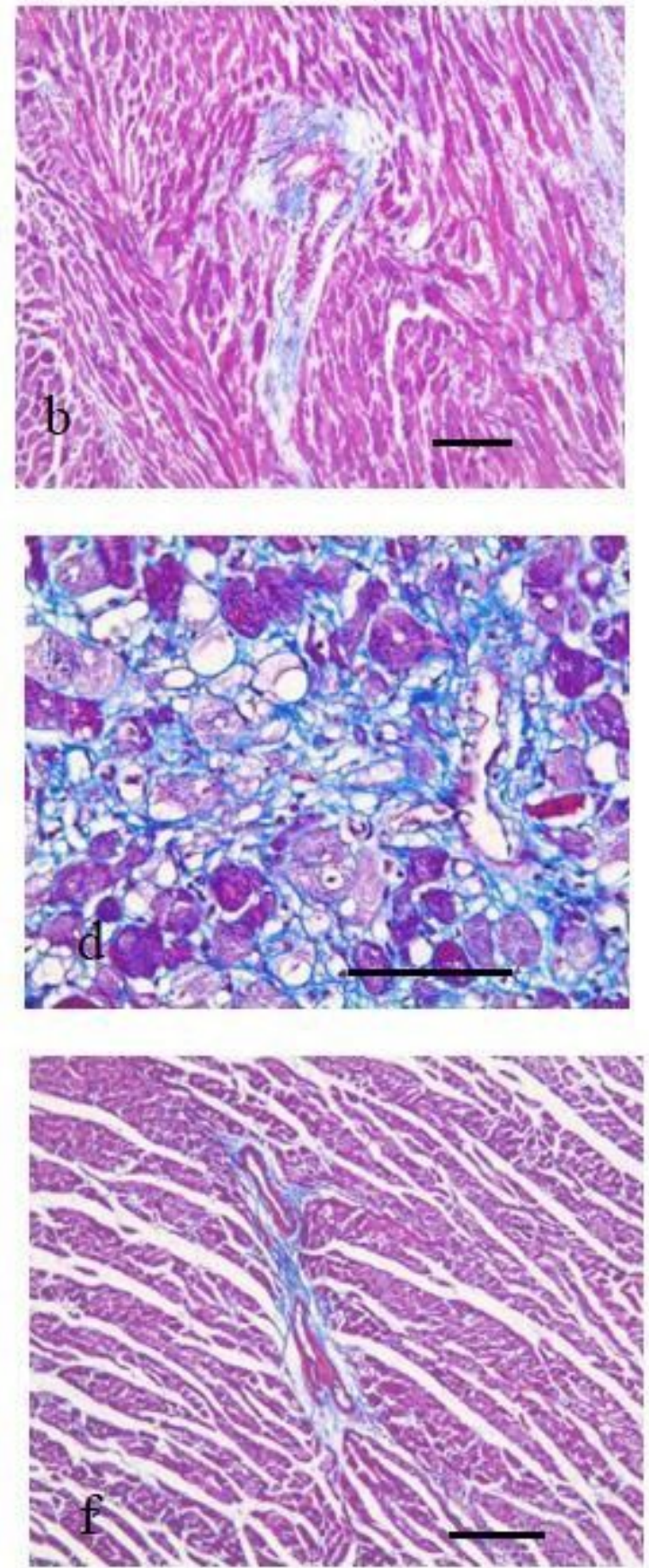

\section{Figure 4}

Masson's staining of heart tissues derived from the normal control (a-b), the DCM control (c-d) and the exosomes group (e-f).Black bars $=50 \mu \mathrm{m}$. 

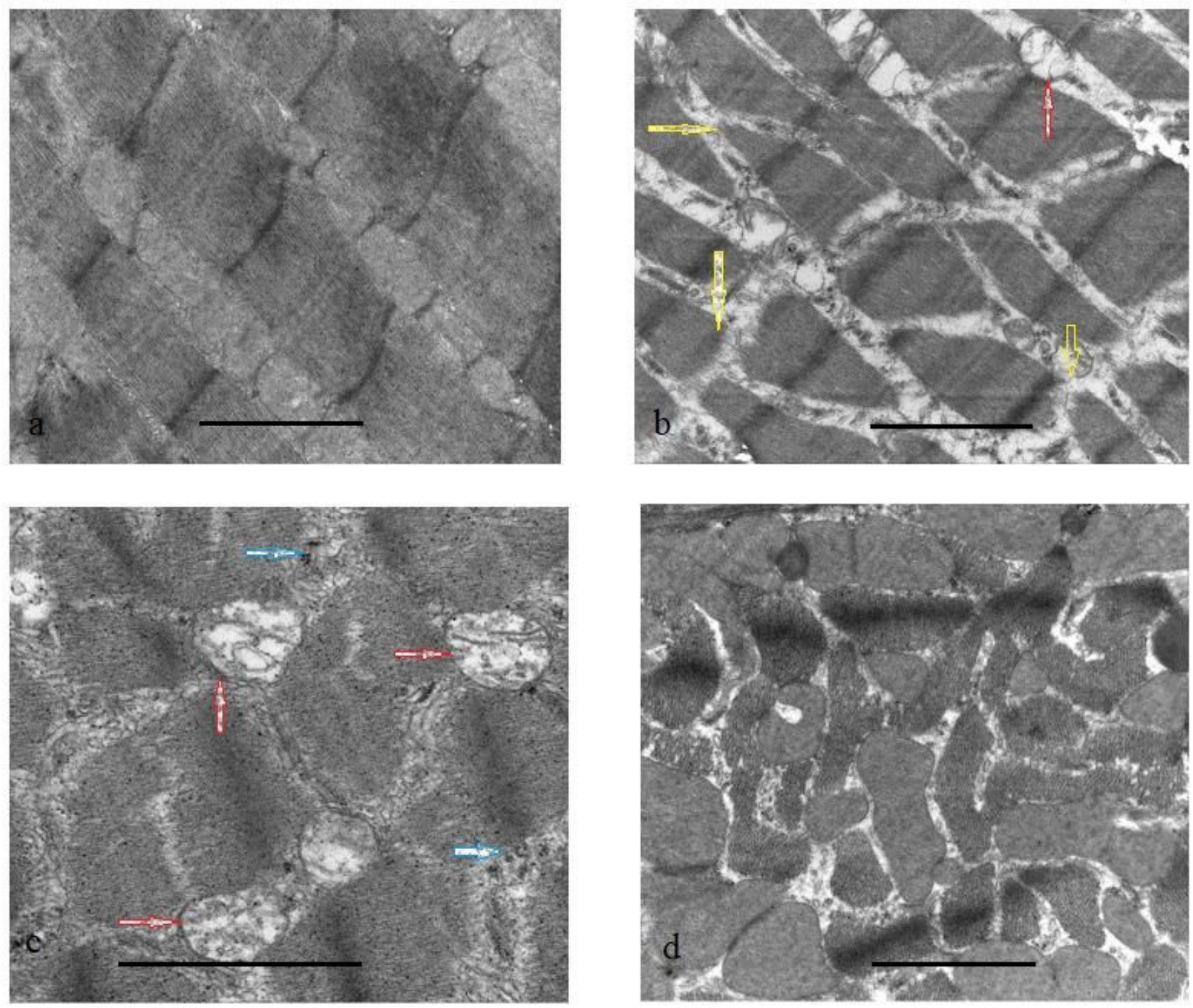

\section{Figure 5}

Transmission electron microscopy images of heart tissues derived from the normal control (a), the DCM control (b-c) and theexosomes group (d). The yellow arrows show the chaotic arrangement of the sparse myofibrils; the red arrowheads show the swelling of mitochondria; the blue arrowheads show glycogen deposits. Black bars $=1 \mu \mathrm{m}$. 

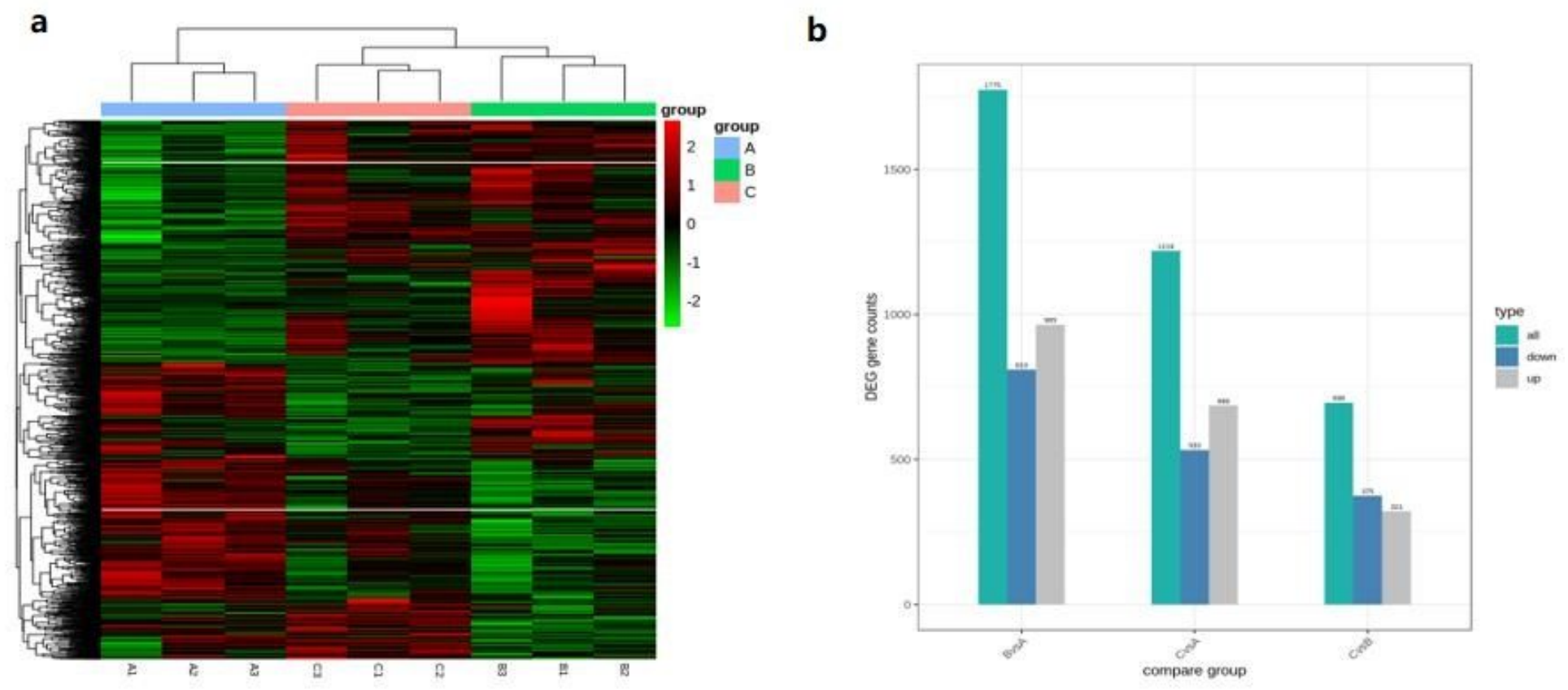

\section{Figure 6}

7 (a) In the heatmap of clustering, the horizontal comparison showed that the expression of the same gene in different groups was different, with red indicating increased expression and green indicating decreased expression. (b) The number of up-regulated and down-regulated genes compared between the two groups. 

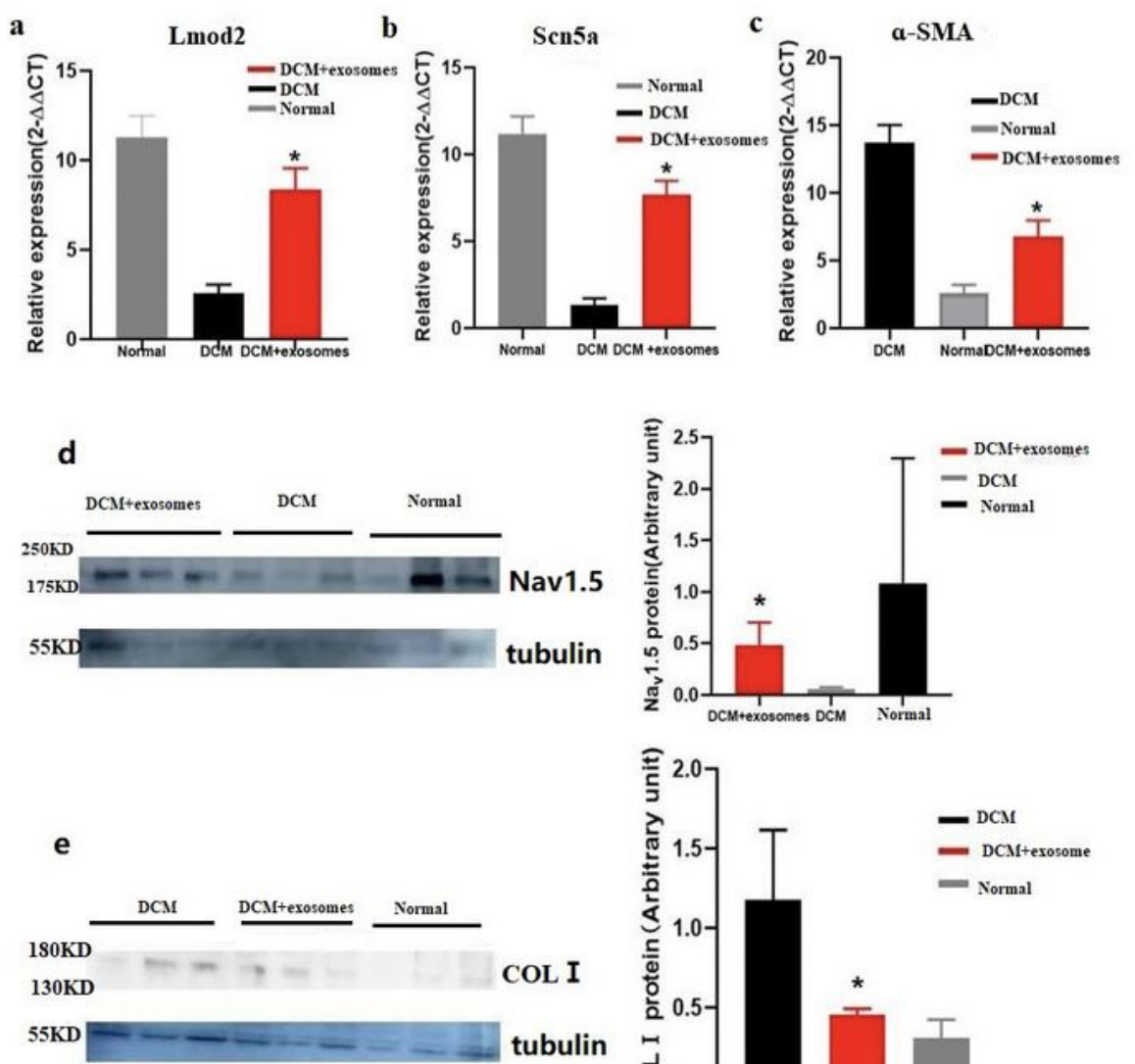

COL I tubulin
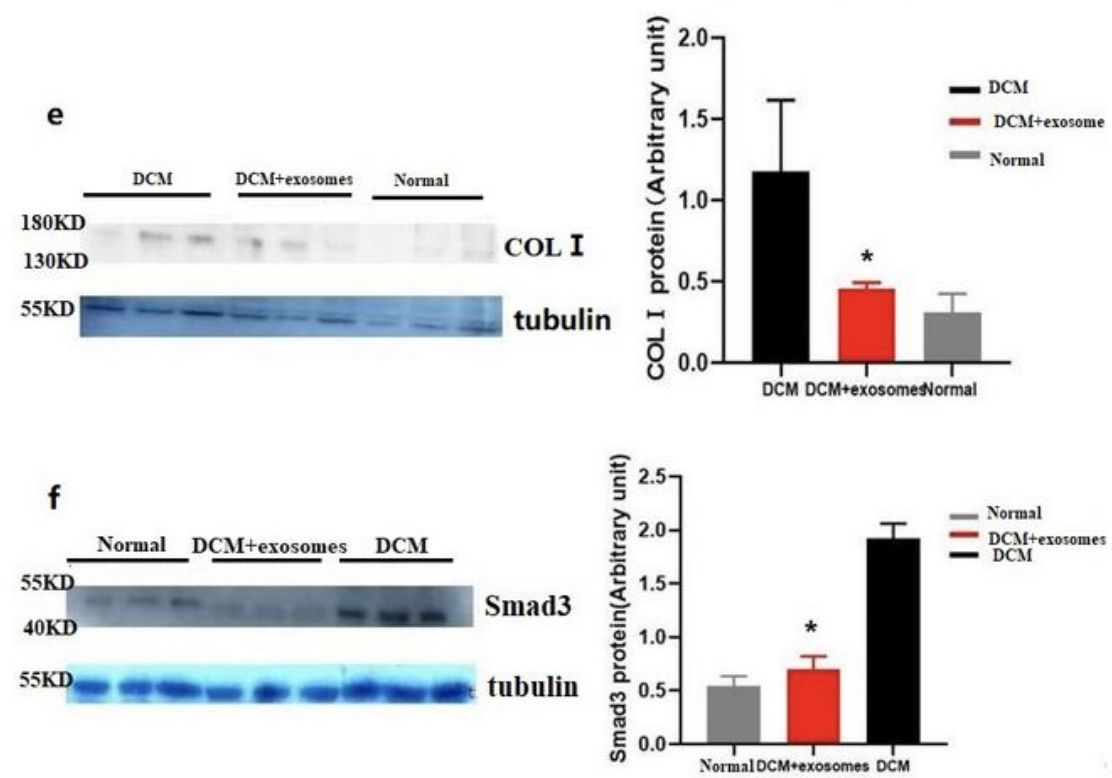

g
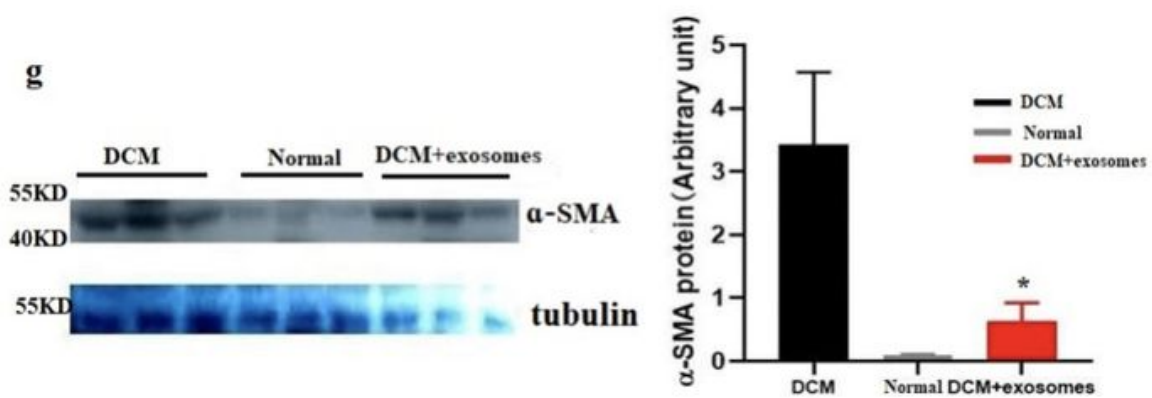

\section{Figure 7}

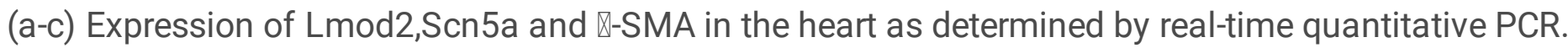
Asterisks represent the statistical difference compared with the DCM group $(P<0.05$, ANOVA). (d-g) A representative figure showing the expression of Nav1.5,COL I,Smad3 and $\mathbb{-}$-SMA in the heart detected by Western blot. Tubulin was detected as a loading control. Asterisks represent the statistical difference compared with the DCM group $(\mathrm{P}<0.05$, ANOVA). 


\section{Supplementary Files}

This is a list of supplementary files associated with this preprint. Click to download.

- SupplementaryMaterial.doc 